\title{
Current Topics
}

\section{Recent Advances in Research on the Mechanisms and Regulation of Allergic Diseases}

\author{
Foreword \\ Takeshi Nabe \\ Laboratory of Immunopharmacology, Faculty of Pharmaceutical Sciences, Setsunan University; \\ Osaka 573-0101, Japan.
}

The numbers of patients suffering allergic diseases such as pollinosis (allergic rhinoconjunctivitis), asthma, and atopic dermatitis have been increasing. Thus, the regulation of allergic diseases has become an urgent task for Japan. Various therapeutic drugs have been developed. For example, in the 1980 s, antiallergic drugs, the so-called mast cell stabilizers, were developed and marketed in Japan, although they were not sufficiently effective, especially for chronic and/or severe allergic diseases. The 1990 s saw the development and marketing of receptor antagonists against cysteinyl leukotrienes and thromboxane $\mathrm{A}_{2}$. Those antagonists exhibited effectiveness in the treatment of some cases of asthma and allergic rhinitis.

At present, according to guidelines on the treatment of allergic diseases, pharmacotherapies largely depend on steroidal antiinflammatory agents, glucocorticoids. Other drugs including antihistamines are additionally used in patients with pollinosis and atopic dermatitis. For asthma therapy, treatment regimens including adrenergic $\beta_{2}$ receptor agonists are used. When appropriately used topically at low doses, glucocorticoids are safe and effective. However, other effective medications in addition to glucocorticoids are required. It has recently been suggested that $5-10 \%$ of asthma patients are unresponsive to glucocorticoid therapy. Steroid resistance appears to be associated with the pathogenesis of chronic/severe asthma.

For the development of clinically useful pharmacotherapies for allergic diseases, it is necessary to elucidate the underlying disease mechanisms. The immunoglobulin E (IgE)-mast cell axis and T-helper 2 (Th2) cell-eosinophil axis have been studied extensively. Research on IgE antibodies led to the development of an anti-IgE antibody formulation that has been shown to be effective in the treatment of severe asthma. Although conflicting reports on the roles of eosinophils in allergic disease were published, it is now recognized that anti-interleukin (IL)-5 antibody and anti-IL5R $\alpha$ antibody are effective in treating asthma. In addition, an anti-IL-4 antibody agent is now available for atopic dermatitis and asthma patients.

Recent research has focused on mechanisms other than the IgE-mast cell and Th2 cell-eosinophil axes. Type 2 inflammation is induced not only by Th2 cells but also by type 2 innate lymphoid cells (ILC2), which are capable of producing high levels of IL-4 and IL-5 in response to various cytokines such as epithelial cell-derived thymic stromal lymphopoietin and IL-33. It was suggested that ILC2 are involved in steroid resistance in asthma. On the one hand, neutrophils have been regarded as cells with a minor role in allergic diseases. On the other hand, neutrophils are known to exist in airway tissues, especially in severe asthma patients. Experimental studies elucidating novel and essential mechanisms are expected to lead to development of new pharmacotherapies for allergic diseases.

As the sole curative therapy, allergen immunotherapy is administered to allergic disease patients, especially via the subcutaneous and sublingual routes for those with pollinosis. Formulations of allergen extracts have recently improved. However, the mechanisms underlying the effectiveness of allergen immunotherapy remain to be clarified, although increases in $\mathrm{IgG} 4$ and regulatory $\mathrm{T}$ cells have been suggested to play important roles. Elucidation of the mechanisms of action of allergen immunotherapy may lead to novel treatments for allergic disease.

In this Current Topics section, four allergy researchers were invited to write on recent advances in research on the mechanisms and regulation of allergic diseases. Dr. Masanori Fujii summarizes the current understanding of the pathophysiological mechanisms of atopic dermatitis. The multiple biological roles of eosinophils in the host defense system, homeostasis, and eosinophil-associated diseases including allergy are described by Dr. Akira Kanda. My report focuses on steroidresistant asthma and the possible roles played by neutrophils in its development. Professor Osamu Kaminuma introduces a novel murine model of $\mathrm{T}$ cell-mediated nasal hyperresponsiveness developed by his group. The process leading to the development of sublingual immunotherapy tablets for allergic rhinitis is the topic of the article contributed by Dr. Katsuyo Ohashi-Doi.

These articles provide a better understanding of the mechanisms underlying allergic disease and will lead to further discoveries of potential therapeutic targets. As the editor of this Current Topics, I sincerely thank all the authors for their valuable contributions. 